vii

\title{
Elementary Proof of a Theorem about Maxima and Minima of $f(x)$.
}

The theorem in question applies to a function $f(x)$ which, together with its derivatives, is continuous in the neighbourhood of the maximum or minimum value.

It may be stated thus: If $f(a)$ is a maximum (minimum) value of $f(x)$, then of the functions $f^{\prime}(a), f^{\prime \prime}(a), f^{\prime \prime \prime}(a), \ldots$ the first that does not vanish must be of even order and negative (positive).....(1)

Take first the case of a maximum value. $f(a)$ is a maximum value of $f(x)$, if the values of $f(x)$ when $x$ is sufficiently near to $a$ are less than $f(a)$; i.e. if a positive number $k$ can be assigned such that $f(a)-f(a \pm h)>0$ when $|h| \ngtr k$, or, as we may express it, when $f(x)$ is increasing just before and decreasing just after $x=a$.

It follows that $f^{\prime}(x)$ must be positive just before and negative just after $x$ passes through the value $a$.

Hence if $f^{\prime}(x)$ is continuous near $x=a$, it must be zero at $x=a$.

Hence $f^{\prime}(x)$ is decreasing just before and just after $x=a$.

Hence $f^{\prime \prime}(x)$ is negative just before and just after $x=a$.

Hence if $f^{\prime \prime}(x)$ is continuous near $x=a, f^{\prime \prime}(a)$ is negative or zero.

But if $f^{\prime \prime}(a)=0$, since $f^{\prime \prime}(x)$ is negative just before and just after $x=a, f^{\prime \prime}(a)$, must be a maximum of $f^{\prime \prime}(x)$.

Thus we conclude that the necessary conditions for $f(a)$ being a maximum value are that $f^{\prime}(a)=0$ and $f^{\prime \prime}(a)$ either negative, or, zero and itself a maximum value of $f^{\prime \prime}(x)$.

It follows that in the latter case $f^{\prime \prime \prime}(a)=0$, and $f^{\text {iv }}(a)$ must be negative, or zero and a maximum of $f^{\mathrm{iv}}(x)$. Proceeding thus, and excluding the case where $f^{\prime}(a), f^{\prime \prime}(a), f^{\prime \prime \prime}(a), \ldots \ldots$ are all zero, we find the necessary condition for $f(a)$ being a maximum to be that stated in (1).

The converse theorem is that the condition stated in (1) is sufficient to secure that $f(a)$ should be a maximum value.

To prove this we first show that if $f^{\prime}(a)=0$, and $f^{\prime \prime}(a)<0$, then $f(a)$ is a maximum.

(3) For $f^{\prime \prime \prime}(x)$ will be $<0$ just before and just after $x=a$.

(4) $\therefore f^{\prime}(x)$ will be decreasing $\ldots \ldots \ldots \ldots \ldots \ldots \ldots$ 
(5) $\therefore f^{\prime}(x)$ will be $>0$ just before and $<0$ just after $x=a$,

(6) $\therefore f(x)$ will be increasing......., decreasing.........

(7) i.e. $f(a)$ will be a maximum.

Again, if $f^{\prime}(a)=0$ and $f^{\prime \prime}(a)=0$ and a maximum of $f^{\prime \prime}(x)$, then $f^{\prime \prime}(x)$ is necessarily $<f(a)$, i.e. $<0$, in the neighbourhood of $x=\dot{a}$. Hence (3), $(4),(5),(6)$ and (7) apply in this case also.

We conclude that if $f^{\prime \prime}(a)$ is negative or zero and a maximum of $f^{\prime \prime}(x)$, and if $f^{\prime}(a)=0$, then $f(a)$ is a maximum value. ..... (8)

Hence if we suppose $f^{(r)}(a)=0$, for the values $1,2,3, \ldots 2 n-1$ of $r$ and $f^{(2 n)}(a)<0,(8)$ enables us to say that $f^{(2 n-2)}(a)$ is a maximum, and thus that $f^{(2 n-4)}(a)$ is a maximum, and so on, till it is proved that $f(a)$ is a maximum value.

Thus a necessary and sufficient condition for $f(a)$ being a maximum value in the case of a function $f(x)$ which, with those of its derivatives which come into the statement, is continuous in the neighbourhood of $x=a$, is :-

(9) The first of the functions $f^{\prime}(a), f^{\prime \prime}(a), f^{\prime \prime \prime}(a), \ldots$ which does not vanish must be of even order and negative.

To extend the theory to minima.

If $f(a)$ is a minimum of $f(x)$, then $-f(a)$ is a maximum of $-f(x)$. Hence for a minimum value of $f(a)$ we have only to change the word negative into positive in condition (9).

The chief interest of the foregoing lies in the fact that the general form of criterion for a maximum or minimum of $f(x)$ has hitherto been deduced by the aid of Taylor's Theorem. Since questions on maxima and minima are amongst the most interesting and practically valuable applications of the Differential Calculus, it may be useful for teachers to have a proof of the criterion in question not requiring Taylor's Theorem.

R. F. Mutrhead.

\section{A Footnote to Euclid, Book III.}

The following two propositions are converses of Euclid HII., 11 and 12.

Prop. 1. If the circumferences of two circles whose centres are $A$ and $B$ have a common point $C$ lying in the straight line $A B$, then the circles touch each other externally at $C$. 